\title{
TYPE 2 MYOCARDIAL INFARCTION IN ACUTE MEDICAL CARE
}

\author{
K. Sateesh, Stitha Pragna, YSN Raju
}

\section{ABSTRACT:}

Background: Type 2 myocardial infarction (MI) is defined as MI secondary to ischemia due to either increased oxygen demand or decreased supply. It is seen in conditions other than coronary artery disease (CAD) contributes to an imbalance between myocardial oxygen supply and/or demand. Little is known about patient characteristics and clinical outcomes.

Methods: A retrospective analysis was performed in patients who were admitted in acute medical care with symptoms suggestive of myocardial ischemia and enzymatic elevation from January 2015 to December 2015. Patients with slight elevation (above the upper limit of normal) of CK-MB were included in the study and compared the clinical and laboratory profile between men and women.

Results: This survey includes a total of 54 patients, Out of which complete details were available in 41 patients (M:23; F:18). The mean age was 42.61. The common causes of type-II MI were Infectious (M:9 (39.1\%), F:10 (55.6\%) followed by haematological disorders (M:3 (13\%), F:5 (27.7\%). The mean CK-MB was 28.00. The mean CPK was 122.5. There was no hypotension, renal failure at the time of admission. One patient had in-hospital mortality out of all patients, whose primary diagnosis was septicaemia with shock who had normal CPK, elevated CK-MB and elevated NT pro BNP levels. Patients with type-II MI were not referred for coronary interventions and managed conservatively.

Conclusions: There is no significant difference in the risk for type 2 MI between men and women. Clinical suspicion and diagnosis of type $2 \mathrm{MI}$ is crucial in acute medical care setting, as the mortality can be reduced with adequate management of underlying condition. The threshold of biomarker levels should be low (above the upper limit of normal in symptomatic ischemia) to label type 2 MI contrary to the third universal definition of MI.

Key words : Myocardial infarction, Acute Medical Care, Incidence.

Article received on 05 OCT 2016, published on 31 OCT 2016.

K. Sateesh ${ }^{1}$, Stitha pragna ${ }^{2}$, YSN Raju

${ }^{1}$ Senior resident, Cardiology , NIMS, Hyderabad, India.

${ }^{2}$ Junior resident, General medicine, NIMS, Hyderabad, India.

${ }^{3}$ Professor and HOD, General medicine, NIMS, Hyderabad, India.

Corresponding Author: K. Sateesh

Email: $\underline{\text { dr.satishkilli@gmail.com }}$

\section{INTRODUCTION}

Myocardial Infarction (MI) has been considered for long time as a single pathological entity. The increasing knowledge about its pathogenesis, the development of more precise and sensitive serological biomarkers, combined with the introduction of more accurate imaging techniques have allowed us to revolutionize our understanding of the underlying mechanisms ultimately leading to cardiac ischemia and to the following irreversible myocardial injury.

Third universal definition of Myocardial infarction has been released by a Joint ESC/ACCF/AHA/WHF Task Force for the redefinition of MI, which officially introduced for the very first time the concept that MI should be classified by as many as five different types according to the pathogenetic mechanisms causing myocardial ischemia and injury [1].

Type 2 MI is defined as irreversible myocardial injury secondary to an imbalance between myocardial oxygen supply and/or demand due to conditions other than coronary atherosclerosis (e.g. severe anaemia, coronary vasospasm, endothelial dysfunction, toxic effects of endogenous or exogenous catecholamine, shock, respiratory failure, heart failure, tachyarrhythmia including atrial fibrillation, hypertension or hypotension, coronary embolism and cardio toxic substances) [1].

Notably, the diagnosis of type 2 MI remains challenging, wherein the definitive demonstration of supply/demand imbalance which has triggered myocardial ischemia is not reliably identifiable on the basis of clinical symptoms alone, but would require the objective demonstration. An accurate diagnosis is essential for tailoring the treatment according to the underlying causal mechanism.

Most of these mechanical or medical treatments (i.e. revascularization procedures, cholesterol-lowering agents, beta blockers) may not be useful in patients with type 2 MI.

It has also been reported that the overall and cardiac mortality of patients with type 2 MI may be substantially higher than that of patients with type $1 \mathrm{MI}$. 


\section{MATERIALS AND METHODS:}

We retrospectively analysed the data collected from acute medical care unit patients who admitted with noncardiac diseases and complained of ischemic symptoms like chest pain or dyspnoea of sudden onset from January 2015 to December 2015. All patients were evaluated after a detailed history, vital data, systemic examination and appropriate investigations like ECG, 2DEcho, cardiac biomarkers (creatinine phosphokinase (CPK) and CK-MB), creatinine, haemoglobin, blood glucose, $\mathrm{HbA1c}$, procalcitonin.

Routine measurements of CK-MB, as measured by mass assay, were performed in all patients [2,3]. CK-MB Mass assay was performed with the immunochemical method as implemented on the ACS-180 analyzer. Blood samples were routinely collected for the measurement of CK-MB levels at baseline. Serial enzymes assessment was done in 7 patients. All laboratory testing was performed by personnel who were blinded to the patient information and study objectives.

\section{STATISTICAL ANALYSIS:}

Baseline parameters were compared between groups using the Student $t$ test for continuous variables and the chi-square test for categorical variables. Results with a $\mathrm{p}$ value $<0.05$ is considered to be significant.

\section{RESULTS:}

Out of 54 patients, complete details were collected in 41 patients. Mean age of the patients was $42 \pm 18$ years (Table 1). Patients with symptoms of ischemia showed normal CPK levels with elevated CK-MB with mean value of 28 without significant difference among men and women. Serial ECGs were performed in all patients which showed no significant fresh changes from baseline.

Table 1 : Demographic features of the patients

\begin{tabular}{|l|l|}
\hline Parameter & Value \\
\hline Age (years) & $42.61 \pm 18.49$ \\
\hline CK- MB & $28 \pm 0.0$ \\
\hline CREATININE PHOSPHOKINASE & $122.5 \pm 90.7$ \\
\hline
\end{tabular}

Men were more involved in this study than women but the difference was not statistically significant. Baseline characteristics and the underlying diseases were depicted in Table 2.

Table 2: Baseline characteristics and the underlying diseases

\begin{tabular}{|l|l|l|l|}
\hline Parameter & Male & Female & P Value \\
\hline No & 23 & 18 & \\
\hline Hematological & $3(13 \%)$ & $5(27.7 \%)$ & 0.25 \\
\hline Infectious & $9(39.1 \%)$ & $10(55.6 \%)$ & 0.29 \\
\hline Metabolic & $1(4.3 \%)$ & $1(5.6 \%)$ & 0.8 \\
\hline Oncological & $4(17.3 \%)$ & $0(0 \%)$ & 0.03 \\
\hline RVD & $6(26.1 \%)$ & $0(0 \%)$ & 0.004 \\
\hline Rheumatological & $0(0 \%)$ & $2(11.1 \%)$ & 0.13 \\
\hline
\end{tabular}

Most common diseases among men were infectious diseases, retroviral, oncologial, haematological and metabolic diseases. Among women infectious, hematological, rheumatological and metabolic diseases. Out of these Retroviral and oncological diseases had statistically significant difference between men and women. The risk factors between men and women were depicted in (Table 3).

Table 3: Comparison between risk factors among men and women.

\begin{tabular}{|l|l|l|l|}
\hline Parameter & Male & Female & P Value \\
\hline DM & $7(30.4 \%)$ & $7(38.9 \%)$ & 0.57 \\
\hline HTN & $7(30.4 \%)$ & $7(38.9 \%)$ & 0.57 \\
\hline Smoking & $5(21.7 \%)$ & $2(11.1 \%)$ & 0.35 \\
\hline History of CAD & $3(13 \%)$ & $3(16.7 \%)$ & 0.75 \\
\hline Usage of statins & $6(26.1 \%)$ & $7(38.9 \%)$ & 0.38 \\
\hline On antiplatelets & $6(26.1 \%)$ & $6(33.3 \%)$ & 0.62 \\
\hline On Steroids & $0(0 \%)$ & $3(16.7 \%)$ & 0.06 \\
\hline Post CVA & $1(4.3 \%)$ & $0(0 \%)$ & 0.31 \\
\hline RWMA of & $1(4.3 \%)$ & $0(0 \%)$ & 0.31 \\
\hline $\begin{array}{l}\text { Presence } \\
\text { Hypokinesia on } \\
\text { Echo }\end{array}$ & $1(4.3 \%)$ & $2(11.1 \%)$ & 0.43 \\
\hline
\end{tabular}

Among the risk factors statistically, no significant difference was observed between men and women like DM, HTN, past history of CAD, smoking except for patients using steroids which is probably due to high incidence of rheumatological diseases among women.

Laboratory parameters among men and women were depicted in Table 4. Men were more anaemic than 
women but the difference was not statistically significant. There was no significant hypotension, renal dysfunction and left ventricular dysfunction in patients at the time of admission. Women had uncontrolled sugars than men and more sicker which was statistically significant.

Table 4: Comparison of laboratory parameters among men and women.

\begin{tabular}{|l|l|l|l|}
\hline Parameter & Male & Female & p value \\
\hline HB & $8.841 \pm 2.595$ & $9.847 \pm 2.610$ & 0.203 \\
\hline RBS & $\begin{array}{l}112.84 \\
39.67\end{array}$ & $\begin{array}{l}193.8 \\
115.9\end{array}$ & 0.001 \\
& $6.247 \pm 1.092$ & $\begin{array}{l}7.387 \\
2.123\end{array}$ & 0.061 \\
\hline HBA1c & $\begin{array}{l}120.00 \quad \pm \\
18.37\end{array}$ & 0.575 \\
\hline $\begin{array}{l}\text { SYSTOLIC BP } \\
\text { at the time of } \\
\text { admission }\end{array}$ & 12.71 & $56.06 \pm 7.91$ & 0.420 \\
\hline EF (\%) & $53.14 \pm 13.39$ & 56.06 & 0.447 \\
\hline LDH & $877 \pm 730$ & $680 \pm 385$ & 1.027 \\
\hline $\begin{array}{l}\text { SERUM } \\
\text { CREATININE }\end{array}$ & $\begin{array}{l}1.027 \\
0.651\end{array}$ & 0.459 & 1.000 \\
\hline
\end{tabular}

None of the patients required Intra-aortic balloon pump (IABP) or Temporary Pacemaker (TPI) support. One patient had In-hospital mortality out of all patients, whose primary diagnosis was septicaemia with shock who had normal CPK levels, elevated CK-MB and NT pro BNP levels.

\section{DISCUSSION:}

Myocardial infarction remains the leading cause of death around the globe, understanding of the underlying pathophysiological mechanisms have substantially contributed to improve the diagnosis and ameliorate the outcomes. The clear definition of the pathological characteristics of myocardial ischemia and infarction led the way to a revised classification of MI into five different types, which are characterized by a distinctive clinical presentation and also necessitate specific treatments [1]. Type $1 \mathrm{MI}$ is now regarded as an event mainly occurring after complication of an existing coronary atherosclerotic disease, whereas type $2 \mathrm{MI}$ is thought to be secondary to ischemia which develops in the setting of a supply and demand imbalance.

It is important to distinguish between these two types of MI, which include the nearly three-fold higher mortality of type 2 MI [5] and the need to tailor medical treatments according to the different pathogenetic mechanisms. In the most recent publications from the task force, type 2 MI was categorized as a myocardial infarction secondary to an ischemic imbalance between blood supply and myocardial oxygen demand [4,5]. Patients may or may not have atherosclerotic coronary artery disease. Instances of ischemic myocardial injury with necrosis where an imbalance between myocardial oxygen supply or demand occur include coronary endothelial dysfunction, coronary artery spasm, coronary embolism, tachy/brady arrhythmias, anemia, respiratory failure, hypotension, and hypertension with or without left ventricular hypertrophy.

Saa by et al [6] in Denmark studied more than 500 patients with an acute MI. They categorized these patients into the 5 subsets described in the task force documents from 2007 and 2012. These investigators identified patients with an acute MI admitted to the hospital during a 1-year period (2010-2011). Seventy-two percent of the patients had type $1 \mathrm{MI}$ and $26 \%$ had type 2. Type 2 patients were older, more likely to be female, had lower blood Troponin values, and had more comorbidities. In present study patients with symptoms of ischemia had elevated CK-MB not meeting the criteria of third universal definition ( $>3$ times ULN).

We could not find any studies in the literature compared the type $2 \mathrm{MI}$ in men and women. In the present study the difference of type 2 MI between men and women was not statistically significant. Sepsis is the most common cause in both men and women followed by anaemia, retroviral, oncological and metabolic causes.

Gideon Y. Stein et al [7] study showed that anemia is the most common cause followed by sepsis, arrhythmia and post operation. Patients with type $2 \mathrm{MI}$ are considerably older, more often women. They more frequently have a history of coronary revascularization, chronic renal failure, diabetes and lower functional status.

In our study, patients with type 2 MI were less often received secondary preventive treatment such as $\beta$ blockers, statins and antiplatelets. In-hospital mortality was $4.3 \%$ in the present study which is less compared to the previous studies.

In Tomasz Baron et al [8] study showed, the higher age and more frequent comorbidities in patients with type 2 
MI, a higher crude mortality was observed during 1-year follow-up in patients with type 2 vs type $1 \mathrm{MI}$.

Gideon et al [7] study showed In-hospital complications were substantially more frequent and short-term and intermediate mortality rates were near three-fold higher, reaching $13.6 \%$ and $23.9 \%$ at 30 days and 1-year, respectively.

\section{LIMITATIONS:}

There are limitations in our study. We could not perform coronary angiogram to these patients. Long term follow up of all patients could not be done. Serial assessment of enzymes could not be performed in all patients.

\section{CONCLUSIONS:}

There is no significant difference in the risk for type $2 \mathrm{MI}$ between men and women. Clinical suspicion and diagnosis of type 2 myocardial infaction is crucial in acute medical care setting, as the mortality can be reduced with adequate management of underlying condition. The threshold of biomarker levels should be low (above the upper limit of normal in symptomatic ischemia) to label type 2 MI contrary to the third universal definition of MI.

\section{REFERENCES :}

1. Thygesen K, Alpert JS, White HD, Joint ESC/ACCF/AHA/WHF Task Force for the Redefinition of Myocardial Infarction. Universal definition of myocardial infarction. Eur Heart J. 2007;28:2525-2538; Circulation. 2007;116:2634 -2653; J Am Coll Cardiol. 2007;50:2173-2195.

2. Prasad A, Herrmann J. Myocardial infarction due to percutaneous coronary intervention. N Engl J Med 2011;364:453-464.

3. Mair J, Artner-Dworzak E, Dienstl A, Lechleitner P, Morass B, Smidt J, Wagner I et al. Detection of acute myocardial infarction by measurement of mass concentration of creatine kinase-MB. Am J Cardiol. 1991;68:1545-1550.

4. Alpert JS, Thygesen K, Antman E, Bassand JP. Myocardial infarction redefined: A consensus document of The Joint European Society of Cardiology/American College of Cardiology Committee for the redefinition of myocardial infarction. J Am Coll Cardiol. 2000;36(3):959969.

5. Thygesen K, Alpert JS, White HD, et al. Third universal definition of myocardial infarction. Eur Heart J. 2012; 33:2551-2567.

6. Saaby L, Poulsen TS, Hosbond S, et al. Classification of myocardial infarction: frequency and features of Type 2 myocardial infarction. AmJ Med. 2013;126(9):789-797.

7. Gideon Y. Stein, Gabriel Herscovici, Roman Korenfeld, et al. Type-II Myocardial Infarction - Patient Characteristics, Management and Outcomes. PLOS ONE 9(1): e84285. doi:10. 1371, journal.pone.0084285.

8. Tomasz Baron, Kristina Hambraeus, Johan Sundström, David Erlinge, Tomas Jernberg, Bertil Lindahl et al Type 2 myocardial infarction in clinical practice . Heart 2015;101: 101-106. 